\title{
BMJ Open Geographical disparities in access to hospital care in Ontario, Canada: a spatial coverage modelling approach
}

\author{
Erjia Ge (D) , ${ }^{1}$ Min Su, ${ }^{2,3}$ Ruiling Zhao, ${ }^{4}$ Zhiyong Huang, ${ }^{5}$ Yina Shan (D) , \\ Xiaolin Wei (iD ${ }^{3}$
}

To cite: Ge E, Su M, Zhao R, et al. Geographical disparities in access to hospital care in Ontario, Canada: a spatial coverage modelling approach. BMJ Open 2021;11:e041474. doi:10.1136/ bmjopen-2020-041474

- Prepublication history for this paper is available online. To view these files, please visit the journal online (http://dx.doi. org/10.1136/bmjopen-2020041474).

Received 17 June 2020 Revised 12 December 2020 Accepted 14 January 2021

Check for updates

(C) Author(s) (or their employer(s)) 2021. Re-use permitted under CC BY-NC. No commercial re-use. See rights and permissions. Published by BMJ.

For numbered affiliations see end of article.

Correspondence to

Dr Erjia Ge; erjia.ge@utoronto.ca

\section{ABSTRACT}

Objectives Previous studies on geographical disparities in healthcare access have been limited by not accounting for the healthcare provider's capacity, a key determinant of supply and demand relationships.

Design This study proposed a spatial coverage modelling approach to evaluate disparities in hospital care access using Canadian Institute for Health Information data in 2007.

Setting This study focusses on accessibility of inpatient and emergency cares at both levels of individual hospital and the administrative regions of Local Health Integration Network (LHIN) levels.

Measures We integrated a set of traffic and geographical data to precisely estimate travel time as a measure of the level of accessibility to the nearest hospital by three scenarios: walking, driving and a combination of the both. We estimated population coverage rates, using hospital capacities and population in the catchments, as a measure of the level of the healthcare availability. Hospital capacities were calculated based on numbers of medical staff and beds, occupation rates and annual working hours of healthcare providers.

Results We observed significant disparities in hospital capacity, travel time and population coverage rate across the LHINs. This study included 25 teaching and 148 community hospitals. The teaching hospitals had stronger capacities with 489209 inpatient and 130773 emergency patients served in the year, while the population served in community hospitals were 2.64 times higher. Compared with north Ontario, more locations in the south could reach to hospitals within $30 \mathrm{~min}$ irrespective of the travel mode. Additionally, Northern Ontario has higher population coverage rates, for example, with $42.6 \sim 46.9 \%$ for inpatient and 15.7 44\% for emergency cares, compared with $2.4 \sim 34.7 \%$ and $0.35 \sim 14.6 \%$ in Southern Ontario, within a 30 min catchment by driving.

Conclusion Creating a comprehensive, flexible and integrated healthcare system should be considered as an effective approach to improve equity in access to care.

\section{INTRODUCTION}

Healthcare is an important determinant of health and well-being. The 1978 Alma-Ata Declaration created a healthcare revolution that embodied the principles of equity and social justice, with the goal of improving

\section{Strengths and limitations of this study}

- This study uses a spatial coverage modelling approach to assess geographical disparities in access to hospital cares, which is unique in allowing for the healthcare provider's capacities in the assessment.

- This study includes data from all teaching and community hospital data and employs roads, travel speeds and quite a number of geographical data to precisely estimate the travel times as a measure of accessibility for each location to their nearest hospitals.

- Due to lack of data on healthcare service quality, our estimates can only reflect the availability level in terms of the hospital's capacity.

- The estimates for population coverage rates can be more precise if detailed information on population distribution, particularly among people with chronic diseases, is available for the analysis.

universal and equal access to healthcare worldwide. ${ }^{12}$ However, there remain many challenges to achieving 'Health for All'. Health systems play major roles in widening health inequities. According to the inverse care law, the availability of good quality medical care tends to be inversely related to the need for it. ${ }^{3}$

Access to healthcare services is multidimensional. According to Penchansky and Thomas's theory, ${ }^{4}$ there are five dimensions: (1) Accessibility indicates physical distance or travel time between the healthcare providers' site and the user; (2) Availability reflects the opportunity to access the right type of healthcare services when needed; (3) Accommodation refers to the relationships between how supply resources are organised to accept clients and the clients' ability to accommodate these factors and clients' perception of their appropriateness; (4) Affordability describes the relationships between the price of services and the willingness and ability of users to pay for those services, as well as protection from financial consequences 
of health expenses; and (5) Acceptability reflects the responsiveness of healthcare providers to the social and cultural expectations of individual users and communities. Hospitals, clinics and other healthcare facilities are in constant demand regardless of their geographical settings (for example, urban or rural). The location of healthcare providers is vital to ensure that people have access to primary and secondary care, emergency medicine, preventive care, diagnostics and testing, surgery, psychiatry, public health and other types of healthcare. Proximity and access have been shown to have a strong association with service utilisation. ${ }^{5}{ }^{6}$ In this study, we focus on the first two dimensions as a starting point to uncover hospital care access.

In terms of healthcare system performance, the spatial elements of accessibility and availability can be converted to accessibility coverage and availability coverage. Accessibility coverage describes how physically accessible resources are for the population. ${ }^{7}$ Travel time is recommended by the WHO to assess geographical accessibility. For accessibility coverage, the maximum capacity of the service is determined by the number of people who can reach and use the facilities. Availability coverage refers to what resources are available and in what amount the service is delivered. The availability of resources limits the maximum capacity of the service and thus determines the total service that can be provided to the population. Combining accessibility and availability coverages enables us to define spatial coverage, which simultaneously considers the location and maximum coverage capacity of healthcare facilities, the geographical distribution of the population, the landscape through which the patient needs to cross to reach the health facility and the transportation mode. ${ }^{8}$ Further, this method was developed as a standard model, the AccessMod, ${ }^{9}$ supported by WHO and is currently used in the studies of access to emergency hospital care in sub-Saharan Africa, ${ }^{10}$ primary healthcare network in the Western Province of Rwanda ${ }^{11}$ and geographical accessibility to healthcare and malnutrition in Rwanda. ${ }^{12}$

One objective of 2004 Canadian Health Act is "to protect, promote, and restore the physical and mental well-being of Canadian residents and to facilitate reasonable access to health services without financial or other barriers." However, the healthcare system underwent a series of unprecedented changes in the 1990s as the federal government reduced its financial support to the provinces, which caused hospital closures and medical insurance reductions. ${ }^{13}$ There are concerns about whether healthcare access would decline alongside the limited medical resources. ${ }^{14}$ Although the Canadian government continues to implement plans for healthcare reform, a nationwide study indicates that disparities of healthcare access are aggregated in urban areas, especially in socioeconomically disadvantaged neighbourhoods. ${ }^{5}$

In Ontario, hospital care, including inpatient and emergency care, is publicly funded. According to federal legislation, all residents should have reasonable access to these services. ${ }^{15}$ Based on the model of shifting hospital care to primary care, Ontario has cut 18000 hospital beds and staff in the last 30 years and has substantially reduced its funding for public hospitals. ${ }^{16}$ According to Canadian Institute for Health Information (CIHI) data, Ontario has 2.3 hospital beds and 1.4 acute beds per 1000 people. This figure is lower than the average of other provinces with 3.5 beds per 1000 people in 2019. The resource shortage would easily overload Ontario hospitals in densely populated areas where most hospitals regularly operate at $100 \%$ of their capacity or greater, even though the widely accepted standard is around $85 \% .^{17}$ However, variation in supply exists at the Local Health Integration Network (LHIN) level. The numbers of physicians and specialists per 100000 people can vary widely at 53.9 and 127.3, respectively, in low-supply LHINs to 451.8 and 308.0, respectively, in Toronto Central, a high-supply LHIN. $^{18}$

Rickard and his colleagues used travel time to evaluate geographical accessibility of primary care for neighbourhoods in rural and Northern Ontario, where population of residents are less than $30000 .{ }^{19}$ The study found that $97.8 \%$ residents in these neighbourhoods could reach the nearest emergency department in $30 \mathrm{~min}$ by car. However, this result did not consider the supply factor. Green and the team used two-step floating catchment area (2SFCA) method to assess geographical accessibility of primary care physicians in Ontario ${ }^{20}$ by calculating the ratio of healthcare facilities within a catchment area defined by travel time. Although this method has been commonly used in healthcare accessibility studies, ${ }^{21-23}$ it does not take the healthcare provider's capacity into account in the assessment. Additionally, 2SFCA does not provide the estimates for population coverage rate in the predefined catchment area, which is crucial for policymakers. Given on these critical methodological gaps, we proposed a geospatial approach by integrating travel times and population coverages to assess disparities in hospital care access. Specifically, this study aims to (1) explore geographical disparities of travel times to the nearest hospital by walking, driving and the mixture of the both travel modes; and (2) estimate disparities of hospital coverage rates in the North and South Ontario, and across the 14 different LHINs.

\section{METHODS \\ Settings}

In this study, we applied an ecological design. We aggregated hospital and roads and a series geographical data to assess the disparities in access to hospital across multiple scales in geography, including the individual $(250 \times 250$ metres pixel), regional (North and South) and LHINs levels.

\section{Data}

Hospital service capacity

All teaching and community hospitals in Ontario were included in the geospatial accessibility analysis. Locations 


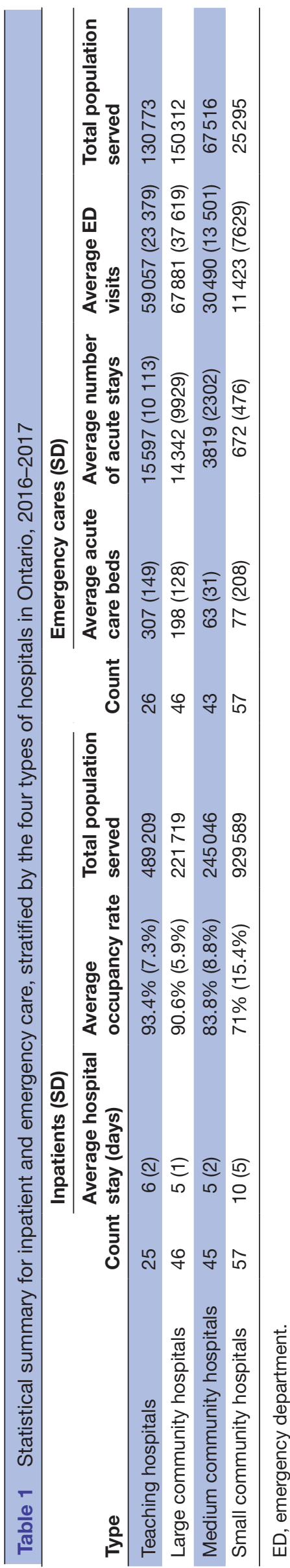

of hospitals, facilities such as beds, number of medical staffs were obtained from the CIHI, an independent not-for-profit organisation that provides information on Canada's health systems and the health of Canadians. Statistics on average hospital stay days, annual occupancy rate, average number of acute stays, average emergency department visits and the total population served by inpatient and emergency cares in 2007 were also available for each hospital from CIHI.

In this study, we categorised hospitals into teaching and community hospitals (also called non-teaching hospitals). Community hospitals were further defined as small, medium or large based on their annual numbers of weighted cases or inpatient days. ${ }^{24}$ A large community hospital has at least 8000 inpatients, more than 10000 weighted cases, or at least 50000 inpatient days per year; a medium community hospital provides care for at least 2000 weighted cases per year; and a small community hospital provides care for less than 2000 weighted cases. We obtained information on hospital location, acute care hospital stays, acute care beds, average time length of hospital stays, bed occupation rate and working days per year from the CIHI in 2016/2017. These data were used to estimate inpatient service capacities. Additionally, we collected information on the number of emergency department visits, medical staff and administrations per capita per year from the CIHI to estimate the capacity of emergency care for each hospital.

\section{Travel time estimates}

We collected roads, streets, maximum speed limits, altitude, rivers, lakes and other land use data from the Ontario Ministry of National Resource and Forestry to estimate travel times between each location and its nearest hospital. Further, we collected $1 \times 1 \mathrm{~km}$ population grid estimate data ${ }^{25}$ from the Centre for International Earth Science Information Network at Columbia University and converted the data into a finer resolution of $250 \times 250$ metres for the estimate of population coverage in predefined catchment areas. Additionally, we collected information about the boundaries of LHINs from the Ontario Ministry of Health and Long-Term Care to assess geographical disparities in population coverage rates between LHINs. All the spatial data were processed using ArcGIS V.10.6.x.

\section{Patient and public involvement}

In this study, we included hospital administrative data in the measure of hospital service capacity. No individual patients included.

\section{Analysis}

Geographical accessibility

In this study, we precisely calculated travel times based on the use of different roads, travel speeds on different land cover and land use classes ${ }^{86}$ for three different travel modes: walking, driving and a mixture of both. In Scenario 1, patients visit the nearest hospital by driving. 


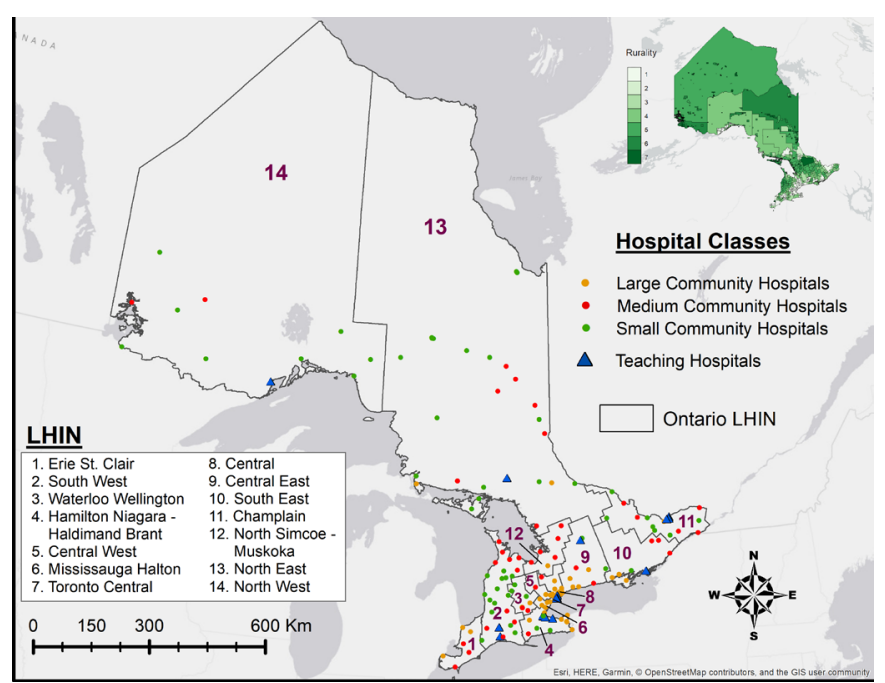

Figure 1 Distribution of hospitals in the 14 LHINs of Ontario, Canada. LHIN, Local Health Integration Network.

This is a predominant form of transportation in rural regions. ${ }^{27}$ The driving speed was determined by maximum road speed limits. Scenario 2 assumes that patients visit the nearest hospital by walking, which is commonly applicable in central or downtown areas. The average walking speed on flat surface was $5 \mathrm{~km}$ per hour as suggested in the modelling. ${ }^{11}$ Scenario 3 considers patients who walk to the nearest parking area and continue travelling with a car. This mixed transportation mode is common for people living in suburban areas. ${ }^{27}$ We set the three maximum travel time limits, which were 30,60 and $120 \mathrm{~min}$. We estimated the geographical accessibility to the nearest hospitals by applying the three predefined maximum travel time limits to each of three travel scenarios.

We created raster surfaces to present the travel times between hospitals and each location point for the three travel scenarios using AccessMod, V.5. ${ }^{9}$ The travel times were estimated using the least-cost path algorithm that accounts for topology and landcover (eg, rivers and lakes), road network and the travel speeds through each road and landcover class. As the topology of terrain may accelerate or impede travelling speeds, the model included altitudes to evaluate the effects of uphill or downward slopes when walking or driving based on Tobler's formula. ${ }^{28}$

\section{Modelling spatial population coverage}

We calculated population coverage rates for each hospital in terms of its location, capacity and catchment population. According to Doherty, ${ }^{29}$ hospital capacities can be measured by the maximum number of patients who can be served. We estimated the maximum number of patients served by inpatient and emergency care, separately, for each hospital in terms of their number of medical staff and beds, occupancy rates and working hours per year using the AccessMod models. ${ }^{9}$ A hospital reaches its maximum capacity when its service cannot cover the population within the catchment. Conversely, a hospital works below its capacity when its service can cover everyone in the catchment. The model uses the least-cost algorithm, in which the location of hospital is selected as an origin and the maximum travel times of 30, 60 and $120 \mathrm{~min}$ are used as the limits for determining the extension of the corresponding catchment area. The model assumes that Ontario is a closed system and patients can only be served by one hospital. ${ }^{9}$ Using this approach, we calculated the population coverage rates of inpatient and emergency care for each hospital with respect to the three travel scenarios.

Lastly, we implemented zonal analysis to assess geographical disparities in population coverages by LHINs, which assumes patients could seek hospital care only within the LHINs where they live. Thus, we calculated the coverage rates for inpatient and emergency care, separately, for each LHIN based on the total population within their jurisdictional boundaries.

\section{RESULTS}

\section{Geographical disparities in estimated travel times}

We included 173 hospitals in the study, which included 25 teaching and 148 community hospitals. Except for one teaching hospital and two community hospitals without emergency care, all hospitals offered both inpatient and emergency care. In 2017, the teaching hospitals served over 489000 inpatient and 130000 emergency cases. The number of inpatient and emergency cases served in community hospitals was 2.64 times larger than that in teaching hospitals (table 1). Geographically, these hospitals are unevenly distributed with teaching and large community hospitals aggregating in south urban LHINs, such as Mississauga Halton, Toronto Central, Central and Central East, and only a few medium and small hospitals in rural LHINs in Northern Ontario (figure 1).

We estimated travel times from each location to their nearest hospital by the transportation modes of driving, walking and the mixture of both within the maximum travel times of 30,60 and $120 \mathrm{~min}$, respectively, in the $250 \times 250$-metre raster maps (figure 2). Regardless of the transportation mode, patients living in Southern Ontario had better geographical accessibility to hospitals. Patients in the west of Southern Ontario had shorter travel times to the nearest hospital than those in the east. Further, the mixture of driving and walking resulted in shorter travel times to the nearest hospital than the other two travel modes (figure 2).

\section{Geographical disparities in hospital coverage rates}

The estimated population coverage rates are presented for inpatient and emergency care, separately, in table 2. We observed that the coverage rates decreased as the catchment areas defined by the maximum travel times increased, regardless of the travel method. For example, the average coverage rates of teaching hospitals for inpatient care decreased from $22.2 \%$ to $8.33 \%$ in the catchments defined by 30 to $120 \mathrm{~min}$ of driving. In contrast to the other three types of hospitals, medium community 

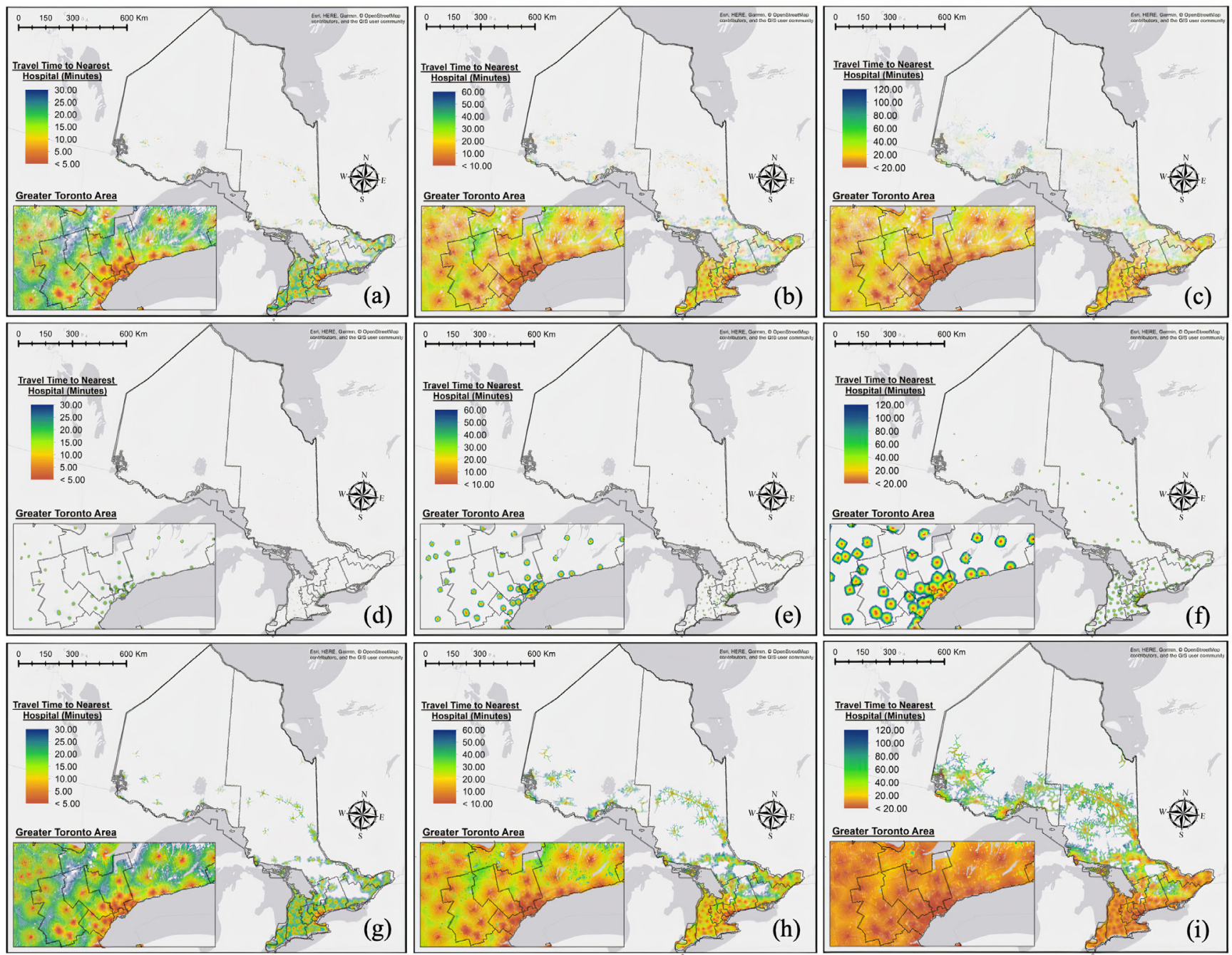

Figure 2 The estimates of travel times to the nearest hospital within the maximum of 30, 60 and 120 min by driving (a, b and $\mathrm{c})$, by walking ( $\mathrm{d}$, e and $\mathrm{f}$ ) and by the mixture of walking and driving ( $\mathrm{g}, \mathrm{h}$ and $\mathrm{i})$.

hospitals' inpatient care tended to have the highest population coverage rates, ranging from $9.53 \%$ to $99.9 \%$, regardless of the catchment size. Compared with inpatient care, emergency care demonstrated a lower coverage rate, except in small community hospitals (table 2).

The zonal analysis results also suggested geographical disparities in population coverage rates between LHINs. We observed higher coverage rates of inpatient care in rural northern LHINs, including North East, North West and North Simcoe Muskoka, but lower rates in urban southern LHINs, including Toronto Central, Central and Mississauga Halton. We observed similar geographical disparities in the coverage rates of emergency care (table 2).

Lastly, the coverage rates calculated based on hospital capacities and the total population of LHIN's jurisdictions are presented in figure 3 . We observed that the coverage rates in northern LHINs were slightly higher than in southern LHINs. The highest coverage rates were found in less densely populated LHINs, such as Champlain in Southeastern Ontario. The inpatient and emergency hospital care offered in Toronto Central LHIN had better population coverages than other urban LHINs.

\section{DISCUSSION}

Many previous studies have contributed important findings on geographical access to healthcare. However, they are less successful in interpreting inequities in healthcare access due to the failure to account for healthcare provider capacity when evaluating the relationship between provider (supply) and population (demand). In this study, we estimated travel times (as a measure of the level of geographical accessibility) to the nearest hospital using three travel modes (walking, driving and a mixture of both), accounting for roads, speed limits and various geographical factors, including elevation, land use, rivers and lakes. We calculated population coverage rates (as a measure of the level of healthcare availability), accounting for hospital locations, capacities and population in catchments. This study is the first to integrate the 


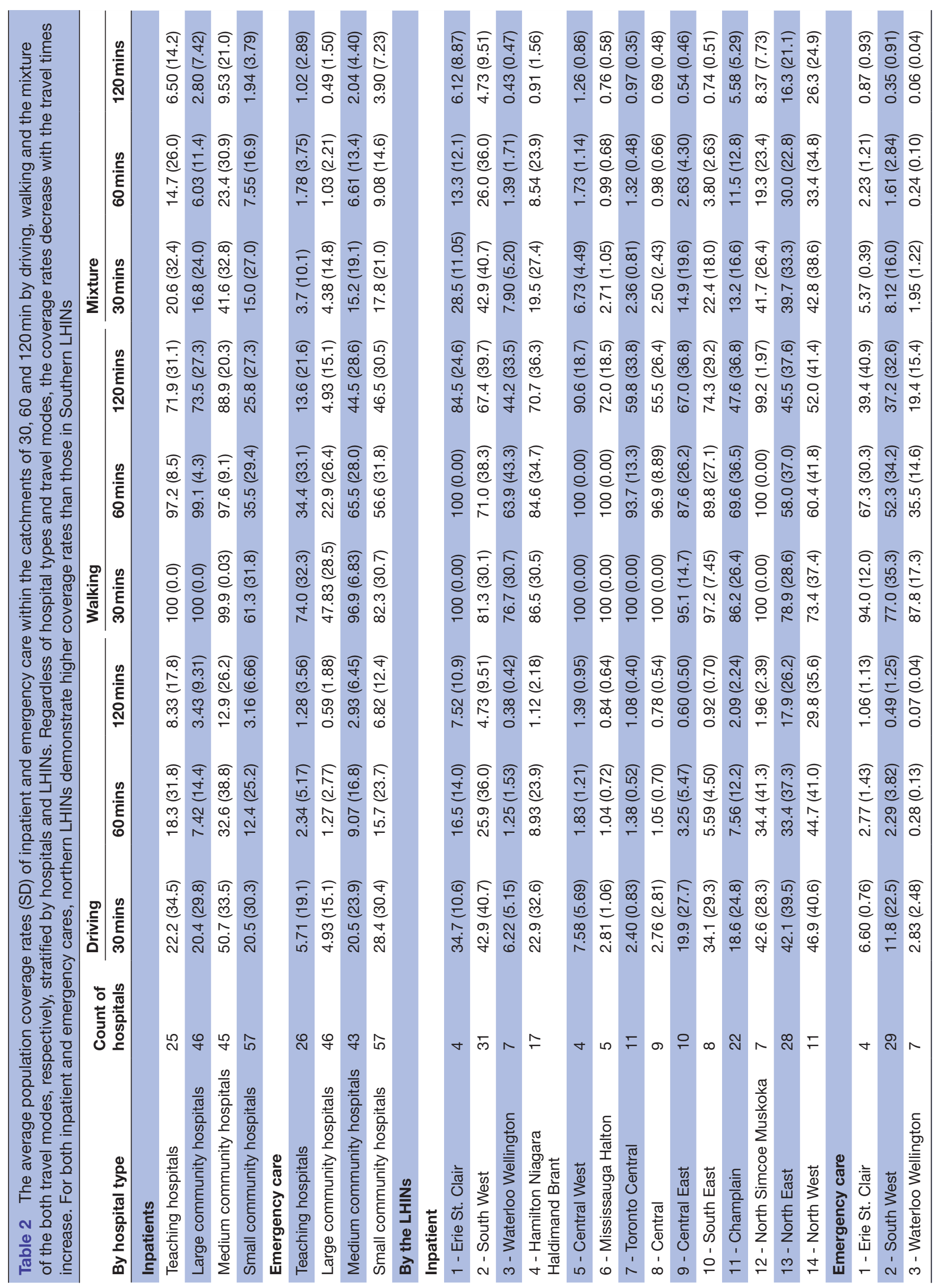


two measures to assess geographical disparities in access to inpatient and emergency care, which may contribute to a more nuanced understanding of the performance of Ontario's current hospital system and the identification of potential gaps.

Although most patients living in Southern Ontario can reach hospitals in $30 \mathrm{~min}$ by car or by walking and driving, most patients in Northern Ontario cannot reach the nearest hospital within $30 \mathrm{~min}$ regardless of travel mode. Further, intra-region disparities exist, wherein marginalised populations, such as patients living in rural areas with poorer transportation and fewer economic resources, have to face the 'double jeopardy' situation. ${ }^{23}$ Launching mobile health clinic buses and satellite facilities may help reduce access inequities in these remote areas.

Additionally, our finding of geographical disparities in the hospital coverage rates (ie, lower coverage rates in urban areas compared with rural areas) indicate disproportionally increasing demands in urban and densely populated areas, even though the vast majority of medical resources have been allocated to teaching and large community hospitals in those areas. Better integration and networking between primary care, home care and different types of healthcare services is in great needs to balance the supply and demand relationship, reduce hospital burdens and improve the efficiency of the use of medical resources. ${ }^{30}$

Lastly, our study proposed an integrated method to precisely measure geographical disparities in access to hospital care. A previous population-based study indicated that over $90 \%$ of people in communities with populations smaller than 30000 could still access the nearest hospital within $30 \mathrm{~min}$ by car. ${ }^{19}$ However, we found that the actual population coverage rates accounting for hospital capacities were much lower, ranging from $16 \%$ to $44 \%$ in Northern LHINs (table 2). The figures were even lower in south urban LHINs, contrary to the previous study. ${ }^{19}$ It should be noted that accessibility to healthcare might be highly overestimated when the healthcare provider's capacity is not accounted for in measure calculations.

This study has several limitations. Although we included details on roads, maximum speed limits, elevations and a series of geographical data to precisely estimate the travel times to the nearest hospital, the measure could not reflect the accessibility levels for some specific groups, such as older adults or persons with disabilities. Additionally, we estimated geographical accessibility to the nearest hospitals by setting three maximum travel time limits, which were 30,60 and $120 \mathrm{~min}$. This estimate could cover most people living in urban, suburban or part of rural areas, however it might not reflect the actual travel time of some specific groups, for example, indigenous or other disadvantage people, who do not drive and live in farther northern rural areas. Although we accounted for hospital capacities in measuring the availability level, no valid data was available for healthcare service quality.

Finally, we estimated population coverage rates based on the general population, which assumed people have 

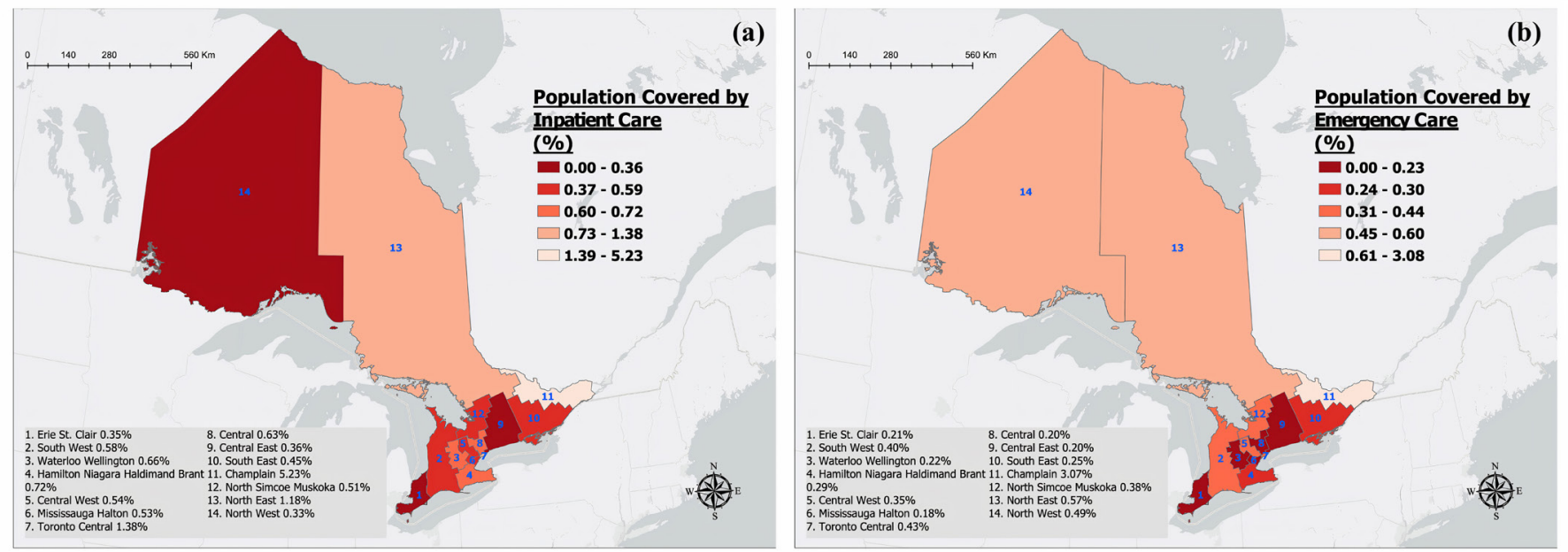

Figure 3 The average population coverage rates of (a) inpatient and (b) emergency cares in the 14 LHINs of Ontario, 20162017. LHIN, Local Health Integration Network.

equal demands for hospital care in their catchments. However, the estimates could be more precise if detailed demographic data were available for the catchments. In future study, non-spatial factors, such as sociocultural barriers, socio-economic disadvantages and healthcare needs, should be included to facilitate a more sound and holistic examination of accessibility in public health and healthcare practice.

\section{CONCLUSION}

The identified significant geographical disparities in hospital capacity, travel time and population coverage rate suggest creating a comprehensive, flexible and integrated healthcare system should be considered to reduce the inequity in healthcare accesses.

\author{
Author affiliations \\ ${ }^{1}$ Divison of Epidemiology, Dalla Lana School of Public Health, University of Toronto, \\ Toronto, Ontario, Canada \\ ${ }^{2}$ School of Public Administration, Inner Mongolia University, Hohhot, Inner Mongolia, \\ China \\ ${ }^{3}$ Division of Clinical Public Health, Institute of Health Policy and Evaluation, \\ University of Toronto, Toronto, Ontario, Canada \\ ${ }^{4}$ Division of Biostatistics, University of Toronto Dalla Lana School of Public Health, \\ Toronto, Ontario, Canada \\ ${ }^{5}$ Department of Mathematics, University of Toronto, Toronto, Ontario, Canada \\ ${ }^{6}$ Health Behavior Science, University of Toronto Dalla Lana School of Public Health, \\ Toronto, Ontario, Canada
}

Acknowledgements We would thank the Canadian Institute for Health Information (CIHI) for the provision of emergency healthcare and hospitalisation data and the Ontario Ministry of National Resource and Forestry (OMNRF) for the provision of roads and geographical data.

Contributors EG, MS and XW designed the study and collected the geographical and health administrative data. $\mathrm{RZ}$ and $\mathrm{ZH}$ conducted geospatial data analyses and modelling. EG, XW and YS interpreted the results and revised the manuscript for important intellectual contents. All authors read, offered interpretation and provided comments on the final draft on the manuscript.

Funding Financial support for this study was provided to $E G, Z H, R Z$ and $Y S$ by the Canadian Institute of Health Research (CIHR) through an Operating Grant: Data Analysis Using Existing Databases and Cohorts - Healthy Cities Intervention Research Grant (DA4-170261, 2020-2022).
Disclaimer The opinions, results and conclusions reported in this paper are those of the authors and are independent from the funding source. The study sponsor had no role in the study design; collection, analysis or interpretation of data; report writing or review; approval of the manuscript; or the decision to submit for publication.

Map disclaimer The depiction of boundaries on this map does not imply the expression of any opinion whatsoever on the part of BMJ (or any member of its group) concerning the legal status of any country, territory, jurisdiction or area or of its authorities. This map is provided without any warranty of any kind, either express or implied.

Competing interests None declared.

Patient and public involvement Patients and/or the public were not involved in the design, or conduct, or reporting, or dissemination plans of this research.

Patient consent for publication Not required.

Ethics approval The study was reviewed and approved by the University of Toronto Health Sciences Research Ethics Board. Informed consent was not required because this study relied on existing public health records rather than direct patient contact, and all health administrative data were aggregated by hospitals and verified by the Canadian Institute for Health Information $(\mathrm{CIHI})$.

Provenance and peer review Not commissioned; externally peer reviewed.

Data availability statement Data may be obtained from a third party and are not publicly available. The hospital service data were obtained from the Canadian Institute for Health Information (CIHI). It could be accessible by reasonable request.

Open access This is an open access article distributed in accordance with the Creative Commons Attribution Non Commercial (CC BY-NC 4.0) license, which permits others to distribute, remix, adapt, build upon this work non-commercially, and license their derivative works on different terms, provided the original work is properly cited, appropriate credit is given, any changes made indicated, and the use is non-commercial. See: http://creativecommons.org/licenses/by-nc/4.0/.

\section{ORCID iDs}

Erjia Ge http://orcid.org/0000-0002-3622-3287

Yina Shan http://orcid.org/0000-0001-7574-6809

Xiaolin Wei http://orcid.org/0000-0002-3076-2650

\section{REFERENCES}

1 WHO. Primary health care : report of the International Conference on Primary Health Care, Alma-Ata, USSR, 6-12 September 1978. World Health Organization 1978. Available: https://apps.who.int/iris/handle/ 10665/39228 [Accessed 28 April 2019].

2 Walley J, Lawn JE, Tinker A, et al. Primary health care: making AlmaAta a reality. Lancet 2008;372:1001-7.

3 Gwatkin DR, Bhuiya A, Victora CG. Making health systems more equitable. The Lancet 2004;364:1273-80. 
4 Penchansky R, Thomas JW. The concept of access: definition and relationship to consumer satisfaction. Med Care 1981;19:127-40.

5 Shah TI, Bell S, Wilson K. Spatial accessibility to health care services: identifying under-Serviced neighbourhoods in Canadian urban areas. PLoS One 2016;11:e0168208.

6 Onega T, Duell EJ, Shi X, et al. Geographic access to cancer care in the U.S. Cancer 2008;112:909-18.

7 Tanahashi T. Health service coverage and its evaluation. Bull World Health Organ 1978;56:295-303.

8 Ray N, Ebener S. AccessMod 3.0: computing geographic coverage and accessibility to health care services using anisotropic movement of patients. Int J Health Geogr 2008;7:63.

9 Ray N, Steeve E, Moser F. AccessMod version 5: Supporting Universal Health Coverage by Modelling Physical Accessibility to Health Care - User Guide \& Tutorial, 2017.

10 Ouma PO, Maina J, Thuranira PN, et al. Access to emergency hospital care provided by the public sector in sub-Saharan Africa in 2015: a geocoded inventory and spatial analysis. Lancet Glob Health 2018;6:e342-50.

11 Huerta Munoz U, Källestål C. Geographical accessibility and spatial coverage modeling of the primary health care network in the Western Province of Rwanda. Int J Health Geogr 2012;11:40.

12 Aoun N, Matsuda H, Sekiyama M. Geographical accessibility to healthcare and malnutrition in Rwanda. Soc Sci Med 2015;130:135-45.

13 James AM. Closing rural hospitals in Saskatchewan: on the road to wellness? Soc Sci Med 1999;49:1021-34.

14 Mendelsohn M. Canadians' Thoughts on Their Health Care System: Preserving the Canadian Model Through Innovation. Queen's University, 2002. Available: http://scholar.googleusercontent.com/ scholar?q=cache:CGlkfNWHD_EJ:scholar.google.com/\&hl=en\&as_ $\mathrm{sdt}=0,5$

15 Canada H. Canada health act. AEM, 2004. Available: https://www. canada.ca/en/health-canada/services/health-care-system/canadahealth-care-system-medicare/canada-health-act.html [Accessed 30 April 2019].

16 Hospital Overload: Ford Government Begins Its Assault on Ontario Hospitals - The Bullet. Socialist Project, 2019. Available: https:// socialistproject.ca/2019/02/hospital-overload/ [Accessed 6 January 2020].

17 Ontario Hospital Association. A balanced approach: the path to ending Hallyway medicine for Ontario patients and families, 2019. Available: https://www.oha.com/Bulletins/A\%20Balanced\% 20Approach\%20-\%202019\%20Pre-Budget\%20Submission.pdf [Accessed 30 January 2020].

18 The Ontario physician human resources data centre (OPHRDC). OPHRDC. Available: http://www.ophrdc.org/ [Accessed 6 January 2020].
19 Rickard HG, Gozdyra P, Yeritsyan N. Geographic Access to Primary Care and Hospital Services for Rural and Northern Communities: Report to the Ontario Ministry of Health and Long-Term Care. Toronto: : Institute for Clinical Evaluation Sciences 2011. Available: https://www.ices.on.ca/Publications/Atlases-and-Reports/2011/ Geographic-Access-to-Primary-Care [Accessed 1 May 2019].

20 Green ME, Gozdyra P, Frymire E. Geographic variation in the supply and distribution of comprehensive primary care physicians in Ontario. Toronto: Institute for Clinical Evaluation Sciences, 2017.

21 Crooks VA, Schuurman N. Interpreting the results of a modified gravity model: examining access to primary health care physicians in five Canadian provinces and territories. BMC Health Serv Res 2012;12:230.

22 Schuurman N, Bérubé M, Crooks VA. Measuring potential spatial access to primary health care physicians using a modified gravity model. The Canadian Geographer / Le Géographe canadien 2010;54:29-45.

23 Wang L. Analysing spatial accessibility to health care: a case study of access by different immigrant groups to primary care physicians in Toronto. Ann GIS 2011;17:237-51.

24 Candian Institute for Health Information. Physicians in Canada, 2016: summary report. Available: https://secure.cihi.ca/free_products/ Physicians in Canada 2016.pdf

25 Gridded Population of the World (GPW), v4, SEDAC. Socioeconomic Data and Applications Center - A Data Ceter in NASA's Earth Oberving System Data and Information System (EOSDIS) - Hosted by CIESIN at Columbia Unviersity. Available: https://sedac.ciesin. columbia.edu/data/collection/gpw-v4 [Accessed 11 January 2020].

26 European Commission Joint Research Centre. Global environment mornitoring unit: travel time to major cities: a global map of accessibility.

27 Noxo Associaties Limited. Improving travel options in small and rural communities. transport Canada, 2009. Available: http://www. octn.ca/uploads/userfiles/files/Improving\%20Travel\%200ptions\% 20in\%20Small\%20and\%20Rural\%20Communities\%20Transport\% 20Canada\%20April\%202009.pdf [Accessed 10 October, 2020].

28 Tobler W. Three presentations on geographical analysis and modeling: non- isotropic geographic modeling; speculations on the geometry of geography. and Global Spatial Analysis 1993 https:// escholarship.org/uc/item/05r820mz

29 Doherty J, Rispel L, Webb N. Developing a plan for primary health care facilities in Soweto, South Africa. Part II: applying locational criteria. Health Policy Plan 1996;11:394-405

30 Canada $\mathrm{H}$. An overview of progress and potential in health system integration in Canada. AEM, 2002. Available: https://www.canada.ca/ en/health-canada/services/health-care-system/reports-publications/ health-care-system/overview-progress-potential-health-systemintegration-canada.html [Accessed 20 December 2019]. 